The conditions (4.1) can be expressed in the form

$$
\left(\tau_{r \theta}\right)_{r=a}=\frac{-2 S_{1}}{\pi} \int_{0}^{\infty} \frac{\sin h t \cos z t}{t} d t
$$

We assume

$$
v=\int_{0}^{\infty} c(t) K_{1}(t r) \cos z t d t
$$

as a solution of the equation of equilibrium (1.3) where $c(t)$ is a function of $t$ only.

The boundary condition (4.2) will be satisfied if

$$
c(t)=\frac{2 S_{1} \sin h t}{t^{2} \pi G K_{2}(t a)}
$$

Therefore

$$
v=\int_{0}^{\infty} \frac{2 S_{1} \sin h t \cos z t K_{1}(t r) d t}{t^{2} \pi G K_{2}(t a)} .
$$

In conclusion I offer my sincere thanks to Dr. B. Sen for his help in the preparation of this paper.

\title{
ON CERTAIN SOLUTIONS OF A PENDULUM-TYPE EQUATION*
}

\section{BY GEORGE SEIFERT (University of Nebraska)}

Introduction. In the study of the oscillations of a synchronous motor around its average angular velocity, a differential equation of the following type, the so-called pendulum-type arises [1]:

$$
\frac{d^{2} \theta}{d t^{2}}+f(\theta) \frac{d \theta}{d t}=g(\theta)
$$

where $f(\theta)$ and $g(\theta)$ are functions of period $2 \pi$ in $\theta$.

It has been shown [2] that in the case where $f(\theta)=\alpha>0$, a constant, and $g(\theta)=$ $\beta-\sin \theta$, where $\beta$ is a constant such that $0<\beta<1$, there exists a constant $\alpha_{c}=$ $\alpha_{c}(\beta)>0$ such that if $\alpha<\alpha_{c}$, eq. (1) will have a solution $\theta(t)$ such that if $\theta^{\prime}(t)=$ $y(\theta)$, then $y(\theta)=y(\theta+2 \pi)$ for all $t$, while if $\alpha \geq \alpha_{c}$, no such solutions exist. Following Vlasov [3] and Minorsky [4], we call any such solution of eq. (1) a periodic solution of the second kind. Physically, such a solution corresponds to a subsynchronous level of performance of the motor described by eq. (1). It is known also [5] that questions of stability of solutions of eq. (1) with respect to the points of equilibrium of (1) involve questions of existence of such solutions.

The purpose of this note is to exhibit a set of explicit conditions on $f(\theta)$ and $g(\theta)$ which insure the existence of periodic solutions of the second kind for (1). Since it has already been noted [5] that if $f(\theta)>0$ and either $g(\theta)<0$ or $g(\theta)>0$ for all $\theta$, there will exist such solutions for eq. (1), we restrict ourselves to the case where $f(\theta)>0$ and the equation $g(\theta)=0$ has a finite number of roots in $0 \leq \theta<2 \pi$.

*Received April 18, 1952. 
We also show (see Remark 1) that in the special case where $f(\theta)=\alpha>0$ and $g(\theta)=$ $\beta-\sin \theta$ where $0<\beta<1$, these conditions lead to a somewhat larger lower bound on $\alpha_{c}$ that was obtained by Tricomi [2].

Theorem. Let $f(\theta)$ and $g(\theta)$ be twice-differentiable functions of period $2 \pi$ in $\theta, f(\theta)$ $>0$, and the roots $\left\{\theta_{i}\right\}, i=0,1, \cdots, 2 n$, of the equation $g(\theta)=0$ in the interval $\theta_{0} \leq \theta \leq \theta_{0}+2 \pi=\theta_{2 n}$ be such that $g^{\prime}\left(\theta_{0}\right)>0$ and $g^{\prime}\left(\theta_{i}\right) \neq 0$. Then eq. (1) will have a periodic solution of the second kind, provided each of the conditions below holds for each integer $j$ such that $0<j \leq n$ :

(i) $-f^{\prime}(\theta) g^{\prime}(\theta)<2 g^{\prime \prime}(\theta)\left(\frac{f^{2}(\theta)}{4}+g^{\prime}(\theta)\right)^{1 / 2}$ for $\theta=\theta_{2 i}$;

$$
f^{\prime}(\theta) g(\theta)<2\left(g^{\prime}\left(\theta_{2 i}\right)-g^{\prime}(\theta)\right)\left(\frac{f^{2}(\theta)}{4}+g^{\prime}\left(\theta_{2 i}\right)\right)^{1 / 2} \quad \text { for } \quad \theta_{2 i-1}<\theta<\theta_{2 i} ;
$$

(iii) the equation $F_{i}(\theta)=f(\theta) h_{j}(\theta)-g(\theta)=0$ has $m \geq 2$ roots $\left\{\rho_{i}^{(i)}\right\}, i=1,2$, $\cdots$, m, such that $\theta_{2 i-2}<\rho_{i}^{(1)}<\rho_{i}^{(2)}<\cdots<\rho_{i}^{(m)}<\theta_{2 i}$, for which $F^{\prime}\left(\rho_{i}^{(i)}\right) \neq 0$ when $i=1,2$, and

$$
\begin{aligned}
\int_{\theta_{j^{*}}}^{\theta_{2 j}} g(\theta) d \theta & \geq \int_{\rho_{i}(2)}^{\theta_{2 i}} f(\theta) h_{j}(\theta) d \theta+h_{j}\left(\rho_{j}^{(2)}\right) \int_{\theta_{j}^{*}}^{\rho_{j}(2)} f(\theta) d \theta \\
h_{j}(\theta) & = \begin{cases}h_{j}^{(1)}(\theta) & \text { for } \quad \theta_{2 i-1}<\theta \leq \theta_{2 i} \\
h_{j}^{(2)}(\theta) & \text { for } \quad \theta_{2 i-2} \leq \theta \leq \theta_{2 i-1},\end{cases} \\
h_{j}^{(1)}(\theta) & =\int_{\theta}^{\theta_{2 i}}\left[\frac{f(s)}{2}+\left(\frac{f^{2}(s)}{4}+g^{\prime}\left(\theta_{2 i}\right)\right)^{1 / 2}\right] d s, \\
h_{j}^{(2)}(\theta) & =\int_{\theta}^{\theta_{2 j}} f(s) d s+h_{i}^{(1)}\left(\theta_{2 i-1}\right),
\end{aligned}
$$

and $\theta_{i}^{*}$ is the largest root of $f(\theta) h_{j}\left(\rho_{i}^{(2)}\right)=g(\theta)$ such that $\theta_{i}^{*} \leq \rho_{i}^{(1)}$

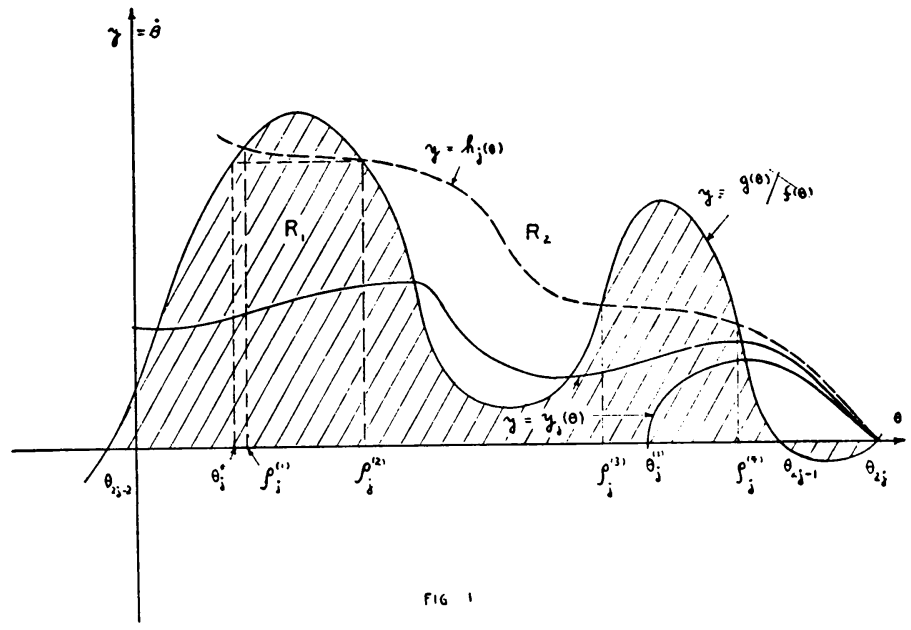


Remark 1. In the case for which $g(\theta)=\beta-\sin \theta, 0<\beta<1$, and $f(\theta)=\alpha>0$, we put $\theta_{1}=\operatorname{arc} \sin \beta, 0<\theta_{1}<\pi_{i} / 2, \theta_{2}=\pi-\theta_{1}$, and $\theta_{0}=\theta_{2}-2 \pi$. Conditions (i) and (ii) of the theorem are trivially satisfied. If we require that

$$
\frac{\alpha}{2}+\left(\frac{\alpha^{2}}{4}+\cos \theta_{1}\right)^{1 / 2} \leq \frac{\beta}{\alpha \theta_{2}},
$$

then $F(\theta)=\alpha h_{1}(\theta)-\beta+\sin \theta=0$ will have two roots, $\rho_{1}^{(1)}<\rho_{1}^{(2)}$ for which $0 \leq \rho_{1}^{(2)}<$ $\theta_{1}$, and clearly $h_{1}\left(\rho_{1}^{(2)}\right) \leq \beta / \alpha$, which implies that $\theta_{1}^{*}$ is such that $\theta_{0}<\theta_{1}^{*} \leq-\pi$. Hence inequality (2) of condition (iii) holds since

$$
\beta\left(\theta_{2}-\theta_{1}^{*}\right)+\cos \theta_{2}-\cos \theta_{1}^{*}>\frac{\beta}{\alpha} \alpha\left(\theta_{2}-\theta_{1}^{*}\right) \geq h_{1}\left(\rho_{1}^{(2)}\right) \alpha\left(\theta_{2}-\theta_{1}^{*}\right),
$$

and thus inequality (3), which can be written as follows:

$$
\alpha \leq \frac{\beta}{\theta_{2}}\left(\frac{\beta}{\theta_{2}}+\cos \theta_{1}\right)^{-1 / 2},
$$

insures the existence of periodic solutions of the second kind in this case. The right hand member of this last inequality will, then, serve as a lower bound on $\alpha_{c}$ defined in the introduction. Tricomi's lower bound on $\alpha_{c}$ is

$$
\frac{\beta}{\pi}\left(\frac{\beta}{\pi}+\cos \theta_{1}\right)^{-1 / 2} \quad[2] .
$$

Proof of the theorem. If we put $d \theta / d t=y$, eq. (1) becomes $d y / d t=g(\theta)-f(\theta) y$, and the equation of the phase trajectories becomes

$$
\frac{d y}{d \theta}=\frac{g(\theta)-f(\theta) y}{y} .
$$

The singularities (in the sense of Poincaré) of eq. (4) consist of the points $\left(\theta_{i}, 0\right)$ of the $(\theta, y)$ cartesian phase plane, the $\theta_{i}$ being the zeros of $g(\theta)$. An analysis of these singularities shows that the points $\left(\theta_{2 i}, 0\right) i=0,1, \cdots, n$, for which $g^{\prime}\left(\theta_{2 i}\right)>0$, are saddle points, and the phase trajectories on these points, the so-called separatrices, have slopes given by

$$
-\frac{f\left(\theta_{2 i}\right)}{2} \pm\left(\frac{f^{2}\left(\theta_{2 i}\right)}{4}+g^{\prime}\left(\theta_{2 i}\right)\right)^{1 / 2}
$$

respectively.

We note that in the region $R_{1}$ of the phase plane bounded by the curve of the equation $y=g(\theta) / f(\theta)$ and the $\theta$-axis all phase trajectories have positive slopes, while in remaining part of the phase plane, $R_{2}$, the trajectories have non-positive slopes, having slope zero only at points on the curve of $y=g(\theta) / f(\theta)$.

For fixed $j$, let us consider the solution $y_{j}(\theta)$ of eq. (4) corresponding to the phase trajectory going into $\left(\theta_{2 i}, 0\right)$ from the left with negative slope; i.e. $y_{i}(\theta)$ is such that

$$
\lim _{\theta \rightarrow \theta_{2 i}-} y_{i}^{\prime}(\theta)=-\frac{f\left(\theta_{2, j}\right)}{2}-\left(\frac{f^{2}\left(\theta_{2 i}\right)}{4}+g^{\prime}\left(\theta_{2 i}\right)\right)^{1 / 2} .
$$

Clearly, we have either $y_{j}(\theta)>0$ for $\theta_{2 i-2}<\theta<\theta_{2 j}$, or there exists a point $\left(\theta_{i}^{(1)}, 0\right)$ such that $\theta_{2 j-2}<\theta_{i}^{(1)}<\theta_{2 j}$ for which $y_{j}\left(\theta_{i}^{(1)}\right)=0$. (See fig. 1). Suppose such a $\theta_{i}^{(1)}$ exists; then the solution $y_{i-1}^{(1)}(\theta)$ for which $y_{i-1}^{(1)}\left(\theta_{2 i-2}\right)=0$ and $\lim _{\theta \rightarrow \theta^{+}, i-}, y_{i-1}^{\prime(1)}(\theta)>0$ 
must be such that $y_{i-1}^{(1)}(\theta)>0$ for $\theta_{2 i-2}<\theta \leq \theta_{2 i}$; for if not, its trajectory would have to intersect the $\theta$ axis in $\theta_{2 i-1} \leq \theta \leq \theta_{2 j}$, which would imply the intersection of the trajectories corresponding to $y_{i-1}^{(1)}(\theta)$ and $y_{i}(\theta)$, which is impossible. Hence $y_{i-1}^{(1)}(\theta)>0$ for $\theta_{2 i-2}<\theta \leq \theta_{2 i}$.

If this holds for $j=1,2, \cdots, n$, it is easily seen that $y_{0}^{(1)}\left(\theta_{2 n}\right)>0$, for if not, its trajectory would have to intersect that of some one of the $y_{j}^{(1)}(\theta), j=1,2, \cdots, n$, which is impossible. Hence, $0=y_{0}^{(1)}\left(\theta_{0}\right)<y_{0}^{(1)}\left(\theta_{2 n}\right)=y_{0}^{(1)}\left(\theta_{0}+2 \pi\right)$. On the other hand, the solution $y_{0}^{(2)}(\theta)$ for which $y_{0}^{(2)}\left(\theta_{0}\right)=M$, where $M$ is such that $g(\theta) / f(\theta)<M$ for all $\theta$, has the property that $y_{0}^{(2)}\left(\theta_{0}\right)>y_{0}^{(2)}\left(\theta_{0}+2 \pi\right)$. By an argument used by Amerio [5], which we omit, we conclude that there exists for eq. (4) a solution such that $y(\theta)=y(\theta+2 \pi)$ for all $\theta$.

We need only show, then, that the existence of some integer $j, 0<j \leq n$, such that $y_{j}(\theta)>0$ for $\theta_{2 j-2}<\theta<\theta_{2 j}$ will contradict the conditions of the theorem. To this end, we show that $y_{i}(\theta)<h_{j}(\theta)$ for $\rho_{i}^{(2)} \leq \theta<\theta_{2 j}$, and $y_{j}(\theta)<h_{i}\left(\rho_{i}^{(2)}\right)$ for $\theta^{*} \leq \theta<\rho_{j}^{(2)}$ where $\rho_{i}^{(2)}$ and $\theta^{*}$ are defined in the statement of the theorem.

We first note that $\theta_{2 j-2}<\rho_{i}^{(2)}<\theta_{2 j-1}$. Define $\phi_{i}(\theta)=h_{i}(\theta)-y_{i}(\theta)$; we have, clearly $\left.\phi_{i}^{\prime}(\theta)_{2,}\right)=\phi_{i}\left(\theta_{2 i}\right)=0$ and will show that condition (i) implies $\phi_{i}^{\prime \prime}\left(\theta_{2 i}\right)>0$. We have from eq. (4),

$$
\begin{aligned}
y_{i}^{\prime \prime}\left(\theta_{2 j}\right)= & \lim _{\theta \rightarrow \theta_{2} j^{-}}\left\{\frac{-\left[y_{i}^{\prime}(\theta)\right]^{2}-f(\theta) y_{i}^{\prime}(\theta)+g^{\prime}(\theta)}{y_{i}(\theta)}-f^{\prime}(\theta)\right\} \\
= & \lim _{\theta \rightarrow \theta_{1} j^{-}}\left\{\frac{-2 y_{j}^{\prime}(\theta) y_{j}^{\prime \prime}(\theta)-f(\theta) y_{i}^{\prime \prime}(\theta)-f^{\prime}(\theta) y_{i}^{\prime}(\theta)+g^{\prime \prime}(\theta)}{y_{i}^{\prime}(\theta)}\right\} \\
& \quad-f^{\prime}\left(\theta_{2 i}\right)
\end{aligned}
$$

by L'Hospital's rule. From this and the fact that $h_{i}^{\prime}\left(\theta_{2 j}\right)=y_{:}^{\prime}\left(\theta_{2 i}\right)$, we obtain

$$
y_{i}^{\prime \prime}\left(\theta_{2 i}\right)=\frac{g^{\prime \prime}\left(\theta_{2 i}\right)-2 f^{\prime}\left(\theta_{2 i}\right) h_{j}\left(\theta_{2 i}\right)}{3 h_{j}^{\prime}\left(\theta_{2 i}\right)+f\left(\theta_{2 i}\right)},
$$

and by a routine calculation which we omit we note that the assumption $h_{i}^{\prime \prime}\left(\theta_{2 i}\right) \leq$ $y_{i}^{\prime \prime}\left(\theta_{2 j}\right)$ would contradict condition (i). Hence $\phi_{i}^{\prime \prime}\left(\theta_{2 i}\right)>0$ and there is an $\epsilon>0$ such that for $\theta_{2 i}-\epsilon<\theta<\theta_{2 i}$, we have $\phi_{i}(\theta)>0$; i.e. $h_{i}(\theta)>y_{j}(\theta)$.

We show next that for any $\theta$ such that $\theta_{2 i-1} \leq \theta<\theta_{2 i}$, the assumption $\phi_{i}(\theta)=0$ would contradict condition (ii). For suppose for $\theta=\theta_{a}$ in this interval, $\phi_{i}\left(\theta_{a}\right)=0$; then there exists a $\xi, \theta_{a}<\xi<\theta_{2 i}$, such that $\phi_{i}^{\prime}(\xi)=0$, $\phi_{i}^{\prime \prime}(\xi) \leq 0$. From eq. (4) we have again

and also

$$
y_{i}^{\prime \prime}(\theta)=\frac{-\left[y_{i}^{\prime}(\theta)\right]^{2}-f(\theta) y_{i}^{\prime}(\theta)+g^{\prime}(\theta)}{y_{i}(\theta)}-f^{\prime}(\theta) \text {, }
$$

hence eq. (5) becomes

$$
y_{j}(\theta)=\frac{g(\theta)}{y_{i}^{\prime}(\theta)+f(\theta)}
$$

$$
y_{i}^{\prime \prime}(\theta)=\frac{\left\{-\left[y_{i}^{\prime}(\theta)\right]^{2}-f(\theta) y_{i}^{\prime}(\theta)+g^{\prime}(\theta)\right\}\left(y_{i}^{\prime}(\theta)+f(\theta)\right)}{g(\theta)}-f^{\prime}(\theta) .
$$

If we put $\theta=\xi$ in this last equation, use $h_{j}^{\prime}(\xi)=y_{j}^{\prime}(\xi)$, and recall that $g(\xi)<0$, a routine calculation will again show that the condition $h_{i}^{\prime \prime}(\xi) \leq y_{i}^{\prime \prime}(\xi)$ contradicts condition (ii).

We next consider the interval $\rho_{i}^{(2)} \leq \theta \leq \theta_{2 j-1}$. Recall that in this interval $g(\theta) \geq 0$, the equality being possible only for $\theta=\theta_{2 j-1}$. Note also that $y_{j}^{\prime}(\theta)=-f(\theta)$ for $\theta=\theta_{2 i-1}$. Now if for $\theta=\theta_{b}, \rho_{i}^{(2)} \leq \theta_{b}<\theta_{2 j-1}$, we have $y_{i}\left(\theta_{b}\right)=h_{j}\left(\theta_{b}\right)=h_{i}^{(2)}\left(\theta_{b}\right)$, there exists an 
$\eta$ such that $\theta_{b}<\eta<\theta_{2 i-1}$ for which $y_{i}^{\prime}(\eta)<-f(\eta)$; but this clearly implies

$$
y_{i}(\eta)=\frac{g(\eta)}{y_{i}^{\prime}(\eta)+f(\eta)}<0
$$

which contradicts the assumption $y_{j}(\theta)>0$ for $\theta_{2 i-2}<\theta<\theta_{2 i}$. We thus conclude that $y_{i}(\theta)<h_{i}(\theta)$ for $\rho_{i}^{(2)} \leq \theta \leq \theta_{2 i-1}$.

Finally, we note that for $\theta_{i}^{*} \leq \theta \leq \rho_{i}^{(2)}$, the curve of $y=f(\theta) / g(\theta)$ is above the line $y=h_{j}^{(2)}\left(\rho_{j}^{(2)}\right)$; this clearly implies that $y_{i}(\theta)<h_{i}\left(\rho_{i}^{(2)}\right)$ for $\theta$ in this interval.

To sum up, we have that for $\rho_{j}^{(2)} \leq \theta<\theta_{2 i}$ we have $y_{j}(\theta)<h_{j}(\theta)$, while for $\theta_{i}^{*} \leq$ $\theta \leq \rho_{i}^{(2)}$ we have $y_{i}(\theta)<h_{j}\left(\rho_{j}^{(2)}\right)$.

We now substitute $y=y_{i}(\theta)$ in eq. (4), multiply by $y_{i}(\theta)$, and integrate from $\theta_{i}^{*}$ to $\theta_{2 i} ;$ we obtain

Hence

$$
-\left[y_{j}\left(\theta_{i}^{*}\right)\right]^{2} / 2=\int_{\theta_{1}^{*}}^{\theta_{2 i}} g(\theta) d \theta-\int_{\theta_{i} *}^{\theta_{2 i}} f(\theta) y_{j}(\theta) d \theta .
$$

$$
\int_{\theta_{*}}^{\theta_{2 i}} g(\theta) d \theta<\int_{\theta_{j^{*}}}^{\theta_{2 i}} f(\theta) y_{j}(\theta) d \theta \text {. }
$$

However, clearly,

$$
\int_{\theta_{i^{*}}}^{\theta_{2 i}} f(\theta) y_{j}(\theta) d \theta<\int_{\rho_{i}(2)}^{\theta_{2} j} f(\theta) h_{j}(\theta) d \theta+h_{i}\left(\rho_{j}^{(2)}\right) \int_{\theta_{i^{*}}}^{\rho_{j}(\boldsymbol{s})} f(\theta) d \theta
$$

which, taken with the previous inequality, contradicts condition (iii). This proves the existence of $\theta_{i}^{(1)}$, such that $\theta_{2 j-2} \leq \theta_{i}^{(1)} \leq \theta_{2 i}$, for which $y,\left(\theta_{i}^{(1)}\right)=0$; hence, the theorem.

Remark 2. In the special case considered in Remark 1, we note that

$$
h_{1}^{\prime}(\theta) \$ \frac{\beta-\sin \theta-\alpha h_{1}(\theta)}{h_{1}(\theta)} \text { for } 0<\theta<\theta_{1} .
$$

This shows that $y=h_{1}(\theta)$ is not a curve for which each trajectory crossing it from the right passes below it; if it were such a curve, conditions (i) and (ii) clearly would have been unnecessary in this case.

Remark 3. Since it is usually a question of imposing conditions on the parameters in $f(\theta)$, it is sometimes more convenient to replace inequality (2) of condition (iii) by the simpler but stronger condition:

$$
\int_{\theta_{i^{*}}}^{\theta_{2 i}} g(\theta) d \theta \geq h_{j}\left(\rho_{i}^{(2)}\right) \int_{\theta_{j^{*}}}^{\theta_{2 i}} f(\theta) d \theta .
$$

On the other hand, condition (iii) can clearly, at the expense of simplicity, be weakened. We omit the details.

\section{REFERENCES}

[1] H. E. Edgerton and P. Fourmarier, The pulling-into-step of a salient-pole synchronous motor, Trans. A.I.E.E., 50, June 1931, pp. 769-778.

[2] F. Tricomi, Integrazione di un'equazione differenziale presentatasi in elettrotecnia, Ann. R. Sc. Norm. Sup. di Pisa, 1933, pp. 1-20.

[3] N. Vlasov, Oscillations of a synchronous motor, Journal of Technical Physics, U.S.S.R. (Russian), 9, 1939.

[4] N. Minorsky, Introduction to Non-Linear Mechanics, Part I, J. W. Edwards, Ann Arbor, 1947.

[5] I. Amerio, Studio asintotica de moto di un punto su una linea chiusa, per azione die forza indipendenti dal tempo, Ann. R. Sc. Norm. Sup. di Pisa, 1950, pp. 19-57. 